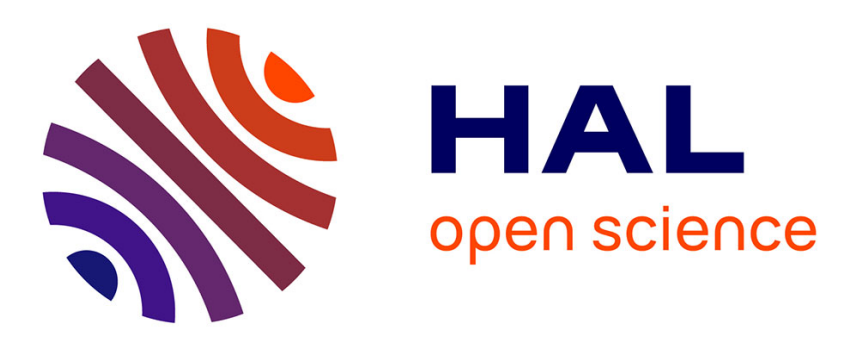

\title{
Interactions digitales et construction identitaire sur les sites Web berbères
}

\author{
Mena B. Lafkioui
}

\section{To cite this version:}

Mena B. Lafkioui. Interactions digitales et construction identitaire sur les sites Web berbères. Études et documents berbères, 2011, 29 - 30, pp.233-253. hal-01116042

\section{HAL Id: hal-01116042 \\ https://hal.science/hal-01116042}

Submitted on 12 Feb 2015

HAL is a multi-disciplinary open access archive for the deposit and dissemination of scientific research documents, whether they are published or not. The documents may come from teaching and research institutions in France or abroad, or from public or private research centers.
L'archive ouverte pluridisciplinaire HAL, est destinée au dépôt et à la diffusion de documents scientifiques de niveau recherche, publiés ou non, émanant des établissements d'enseignement et de recherche français ou étrangers, des laboratoires publics ou privés. 


\title{
INTERACTIONS DIGITALES ET CONSTRUCTION IDENTITAIRE SUR LES SITES WEB BERBERES
}

\author{
par \\ Mena Lafkioui
}

En dépit de la précarité et de la position socio-politique marginale des langues berbères en Afrique du nord et dans la diaspora, une explosion d'expressions culturelles hybrides, tant du point de vue de la forme que du contenu, a eu lieu au cours des dernières années ${ }^{1}$. Maintenant plus que jamais, ces langues fonctionnent comme une source centrale pour la construction et la reconstruction de l'identité collective berbère, un processus dans lequel l'alphabétisation et les médias électroniques jouent un rôle important. L'un des médias les plus accessibles et tout englobant est Internet. Il fournit de nouveaux moyens pour créer de nouveaux discours et contextes, à savoir des interactions électroniques (tout genre d'interaction assistée par ordinateur).

J'examinerai, dans la présente contribution, la façon dont l'identité est (re)construite dans les interactions électroniques sur les sites Web berbères ${ }^{2}$. J'ai opté pour des sites Web à base néerlandaise car ils sont bien développés et régulièrement mis à jour. Ces sites montrent également un niveau élevé de créativité liée à la forte présence de Berbères aux Pays-Bas qui expriment un vif intérêt pour la préservation et la promotion de leur tradition culturelle et de leur identité ethnique. Les sites Web considérés dans cette recherche sont les plus développés (voir le nombre et la conception des liens hypertextes mis à jour, les sous-pages et les items) et les plus populaires (voir nombre de membres inscrits et de hits) : www.amazigh.nl (7446 membres enregistrés le 25/06/08; 7912 sujets forum) et www.tawiza.nl (1255 membres enregistrés le 25/06/08; 2652 sujets forum). Les exemples présentés dans cet article proviennent du site Tawiza (www.tawiza.nl).

Il importe de noter que relativement peu d'attention a été accordée aux problématiques sociolinguistiques et pragmatiques dans la recherche sur la

1 Toutefois, le statut socio-politique des langues berbères est en train de changer en Afrique du nord. Elles ont récemment acquis un statut national - mais non pas «officiel » - au Maroc (1994) et en Algérie (2002). Des instituts nationaux comme le HCA (Haut Commissariat de la Culture Amazighe ; 1995) en Algérie et l'IRCAM (Institut Royal de la Culture Amazighe ; 2001) au Maroc ont été créés afin de maintenir et de développer le patrimoine culturel berbère. L'insertion du berbère dans le programme d'éducation nationale de ces pays est un résultat important de cette évolution, même si ces langues sont encore considérées, selon la constitution nationale des états respectifs, comme des «dialectes » qui servent à améliorer l'acquisition et la maîtrise de l'arabe standard. Cette politique linguistique s'inscrit parfaitement dans les programmes d'enseignement linguistique pour les langues minoritaires existant dans la diaspora.

2 A ce propos, voir aussi Lafkioui (2008a, 2008b). 
communication assistée par ordinateur (Computer Mediated Communication), à l'exception de certaines références notables comme Herring (1993, 1996, 1999), Georgakopoulou (1997, 2003) et Androutsopoulos (2006a/b).

La démarche la plus appropriée pour une connaissance approfondie des processus fondamentaux de la construction identitaire dans un contexte multiculturel et multilingue est la sociolinguistique interactionnelle, principalement en raison de sa conception dynamique de l'identité (Lafkioui, 2008b ; Rampton, 1995 ${ }^{3}$ ). Les identités sont construites et reconstruites en permanence par les interlocuteurs au cours de leurs interactions verbales et non verbales. Elles sont donc des processus sémiotiques interactifs. En ce qui concerne la multiplicité de l'identité (âge, classe, sexe, origine géographique et ethnique, situation géopolitique, statut social, orientation sexuelle, religion) et son hybridité, cette perspective permet l'analyse minutieuse d'un nombre considérable de variables par le biais d'une corrélation entre les variables sociales et les variables linguistiques dans un cadre multidimensionnel ${ }^{4}$.

Cette approche est enrichie par l'utilisation de certaines notions de la perspective sociolinguistique post-structuraliste, qui est fondée sur le modèle interactionnel. C'est le critère de pouvoir (surtout le pouvoir politique) qui est particulièrement important pour la présente étude car le discours en tant que processus social peut être une source d'acquisition et d'exercice du pouvoir, ce qui le rend, par conséquent, essentiel pour la (re)construction des identités par des groupes ou des individus minorés ou opprimés (Bourdieu, 1982, 1991; Encrevé, 1982, 2004 ; Encrevé \& Fornel de, 1983) ${ }^{5}$. Des rapports de pouvoir inégaux peuvent empêcher le «droit à la parole » et conduire à un repositionnement de l'interlocuteur «dominé » dans l'espace interactif et à la reconstruction de son identité (Bourdieu, 1991).

\section{LES SITES WEB BERBÈRES ET LEURS RESSOURCES ÉLECTRONIQUES}

Dans le contexte multilingue des Nord-Africains en Afrique et dans la diaspora, les différentes langues en usage n'ont pas le même statut sociolinguistique ni les mêmes fonctions socioculturelles (Lafkioui, 1998, 2006, 2008a, 2008b). Les politiques nationales et locales déterminent fondamentalement la hiérarchie sociolinguistique des langues employées. De ce fait, aux Pays-Bas, les langues maternelles comme le tarifit (langue berbère de plus de $70 \%$ des Nord-Africains aux Pays-Bas), ayant un statut de «non-officiel » et de «minorité », ne peuvent pas concurrencer avec les langues dominantes : le néerlandais qui est la seule langue officielle aux Pays-Bas et l'anglais qui est généralement considéré comme la langue la plus internationale. Par ailleurs, en dehors du milieu universitaire, aucune formation officielle n'est donnée dans la langue maternelle aux Pays-Bas.

Les internautes visitant les sites Web berbères utilisent principalement le néerlandais et le tarifit (berbère du Maroc du Nord) pour leurs interactions électroniques. Ce sont aussi les deux langues dans lesquelles ces sites sont assemblés. L'arabe (arabe marocain

\footnotetext{
Voir aussi Goffman (1974, 1981, 1983), Gumperz (1982) et Gumperz \& Hymes (1972).

L'approche alternative de socio-psychologie a l'inconvénient de simplifier le rapport entre langue et identité en raison de sa vision unidirectionnelle : elle positionne les actants dans un contexte monolingue et monoculturel qu'elle utilise comme critère de comparaison avec des contextes multilingues (Hamers \& Blanc, 2000 ; Hoffman, 1989 ; Pavlenko, 2002 ; Williams, 1992).

5 Voir aussi Gal (1989), Heller (1988, 1992, 1995a, 1995b) et Blackledge \& Pavlenko (2002).
} 
ou standard), l'anglais et le français sont généralement employés quand un texte écrit ou multimodal est inséré dans le site Web sous son format original. Ainsi, les interactants sont restreints dans leur choix de langue pour leurs interactions électroniques par leur propre répertoire linguistique (compétences linguistiques et interactives) et par le contexte électronique dans lequel les interactions se produisent.

Le contexte électronique englobe à la fois le microcontexte d'une instance d'interaction spécifique dans un cadre temporel bien précis (une interaction de type chat forum par exemple) et le macrocontexte du site Web berbère transnational. Le fait d'avoir accès à ces contextes et, par conséquent, à leurs avantages et restrictions, implique un partage des ressources électroniques que les sites Web sollicitent, en l'occurrence, le néerlandais et le tarifit qui fonctionnent comme ressources linguistiques et interactives.

Le partage de ces ressources entraîne, cependant, l'exclusion d'autres ressources linguistiques, comme l'arabe et le français par exemple, ce qui peut être motivé différemment suivant le programme argumentatif du site 6 . Le tarifit assume essentiellement la fonction de marqueur symbolique de l'identité collective berbère par opposition à d'autres identités collectives locales et translocales, telles par exemple l'identité turque (identité ethnolinguistique locale), hollandaise (identité nationale locale) ou arabo-islamique (identité internationale translocale). Un rôle plus pragmatique et instrumental est attribué au néerlandais comme ressource électronique, bien que cette langue puisse indiquer une identité collective hollandaise par contraste avec d'autres identités nationales, telles que l'identité française sur des sites Web berbères à base française.

Différentes fonctions interactives et catégories sociales sont donc attribuées à différentes ressources, qu'elles soient activées ou non activées dans des contextes en ligne ou hors ligne. Les structures et les fonctions des ressources électroniques sont stratifiées et hiérarchisées (Hymes, 1966, 1996 ; Fairclough, 1992 ; Blommaert, 2005) et, par conséquent, les identités - considérées comme des productions sémiotiques interactives - le sont aussi.

Internet, comme instrument de la mondialisation, permet de remplir les fonctions des ressources linguistiques translocalement et de les repositionner dans l'espace interactif (espace substantiel et cognitif). Les langues berbères qui, en Afrique du nord, sont toujours officiellement perçues comme des «dialectes» de «minorités » à statut socioculturel bas peuvent gagner en prestige à travers le transfert translocal par Internet. Au niveau translocal, ces langues peuvent symboliser des valeurs culturelles hautement estimées et peuvent être inscrites dans des contextes locaux spécifiques (diaspora berbère aux Pays-Bas par exemple) pour créer des identités collectives spécifiques. Internet peut en outre améliorer leur flexibilité sémiotique car, par exemple, la même phrase peut être utilisée dans différents contextes en ligne composés de diverses positions interactives à partir desquelles des identités variées peuvent être construites.

\footnotetext{
${ }^{6}$ J'entends par programme argumentatif l'ensemble des objectifs et fonctions interactifs du site Web.
} 


\section{CONSTRUCTION IDENTITAIRE PAR RECONSTRUCTION ÉLECTRONIQUE DES GENRES ORAUX}

Le caractère oral des langues berbères est en train de changer rapidement en Afrique du nord et dans la diaspora, principalement en raison de la présence de l'écrit et des médias électroniques (Lafkioui, 2008a, 2008b ; Lafkioui \& Merolla, 2008; Merolla, 2005, 2008). Ces derniers ont sérieusement modifié la poésie et la narration orales - tant du point de vue de la forme que du contenu - qui symbolisent les savoirs et les valeurs berbères soigneusement préservés et transmis depuis des siècles en Afrique du nord.

Mon intérêt portera, dans cette section, sur la manière dont certains genres et productions littéraires de la tradition orale berbère sont utilisés pour construire et revendiquer des identités berbères translocales.

Le bilinguisme ou multilinguisme est un aspect important de ce processus de création identitaire. D'une part, il élargit le potentiel sémiotique et, de ce fait, la capacité interactive des interactants. D'autre part, il est un indicateur important de la façon dont les interactants se projettent dans leur discours digital (Lafkioui 2008a).

\section{RECONSTRUCTION DIGITALE DE LA NARRATION ORALE}

Je présenterai, dans ce qui suit, un cas de reconstruction de la narration orale berbère par le biais d'Internet (Lafkioui, 2008a, 2008b). Je montrerai comment le transfert translocal d'un texte oral moyennant Internet peut profondément affecter sa nature, sa structure et parfois même ses fonctions.

Exemple (01)

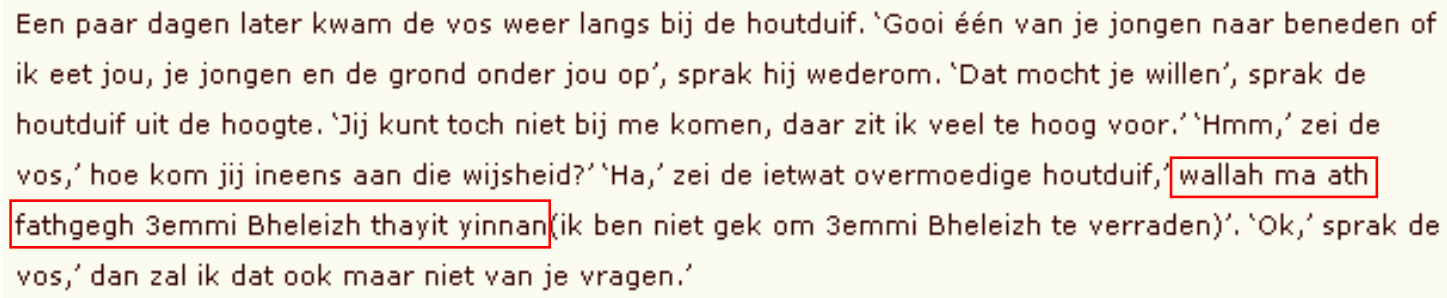

Tmazight - DV, 11-09-2003 (20.42) sur Tawiza

(http://www.tawiza.nl/content/awid.php?id=87\&sid=1\&andra=artikel)

Cet exemple est un extrait d'un texte narratif écrit provenant du site Web berbère à base néerlandaise Tawiza. Ce texte «re-raconte », en néerlandais principalement, un conte berbère traditionnel que l'auteur appelle Oom Ooievaar (néerlandais) ou 3emmi Bheleizh (rifain), ce qui signifie 'Oncle Cigogne'. 
Du fait qu'il fournit le dévoilement de la narration, l'énoncé wallah ma ath fathgegh 3emmi Bheleizh thayit yinnan ('Par Dieu, je ne le dénoncerai pas, Oncle Cigogne me l'a dit !') est emphatisé par son expression en rifain ; autrement dit, l'alternance de code entre le rifain et le néerlandais produit l'effet d'emphase. Outre la fonction de contraste narratif, cet énoncé assume également une fonction emblématique et expressive par le biais de sa structure morphosyntaxique bien déterminée (sermon expressif $=$ formule de sermon + négation; voir Lafkioui, 1996) et de son intonation expressive qui n'est cependant pas représentée dans la notation.

L'expression tfoe est un marqueur d'oralité intéressant car il représente littéralement l'acte de cracher, d'habitude réalisé de manière orale (verbalement et non verbalement) dans les interactions berbères.

Les parties en néerlandais révèlent également une dimension orale, principalement indiquée par leur style direct et simple et par la forte présence des locutions brèves et expressives.

Les contes berbères traditionnels sont généralement narrés par des femmes, souvent d'un certain âge. Selon la croyance ancestrale berbère, il est permis de les raconter seulement la nuit, si l'on ne veut pas infliger l'audience de calvitie par leur pouvoir magique. Bien que cette croyance ait perdu son sens pour les jeunes générations de Berbères, elle remplit toujours encore la fonction de contexte d'interaction, un cadre ritualisé bien défini dans lequel des narrations anciennes aussi bien que contemporaines sont localisées.

Le conte digital de 3emmi Bheleizh montre donc comment la narration orale originale, produite en rifain dans des contextes hors ligne bien spécifiques, a été radicalement reconstruite afin de répondre aux critères linguistiques et interactifs que le site Web Tawiza requiert. Ce dernier fonctionne ainsi comme une sorte de système de conventions de contextualisation («contextualisation conventions » de Gumperz, 1992). La recontextualisation digitale du conte oral (reframing) engage différentes dimensions de l'interaction (linguistique, sociale, culturelle et historique), toutes synchronisées dans une seule fonction signifiée, le conte digital. La recontextualisation digitale a changé la capacité créative de la narration orale, mais non pas sans altérer les catégories sociales et culturelles associées avec elle. Comparée au texte original - étant généralement considéré comme normatif -, la version digitale pourrait être perçue comme non littéraire, non authentique, non berbère, ou non adaptée aux présupposés linguistiques et culturels concernant la forme et la fonction du conte berbère ( « wrong-footed ${ }^{7}$ ).

\section{RECONSTRUCTION DIGITALE DES DEVINETTES ORALES}

Les sites Web berbères à base néerlandaise offrent généralement, sous l'Hyperlink «Taal » ('langue'), des devinettes et expressions idiomatiques «traditionnelles » en rifain, écrites en notation latine et traduites en néerlandais.

Le bilinguisme de la composition des devinettes, extraites du site Web Tawiza et présentées ci-dessous dans l'exemple (02), dévoile différentes fonctions et valeurs que les éditeurs/concepteurs du site ont allouées au tarifit et au néerlandais.

Voir Goffman (1974) pour le concept complexe de «footing » qui réfère à la fois à la notion de présupposé et de position interactive. 
Exemple (02)

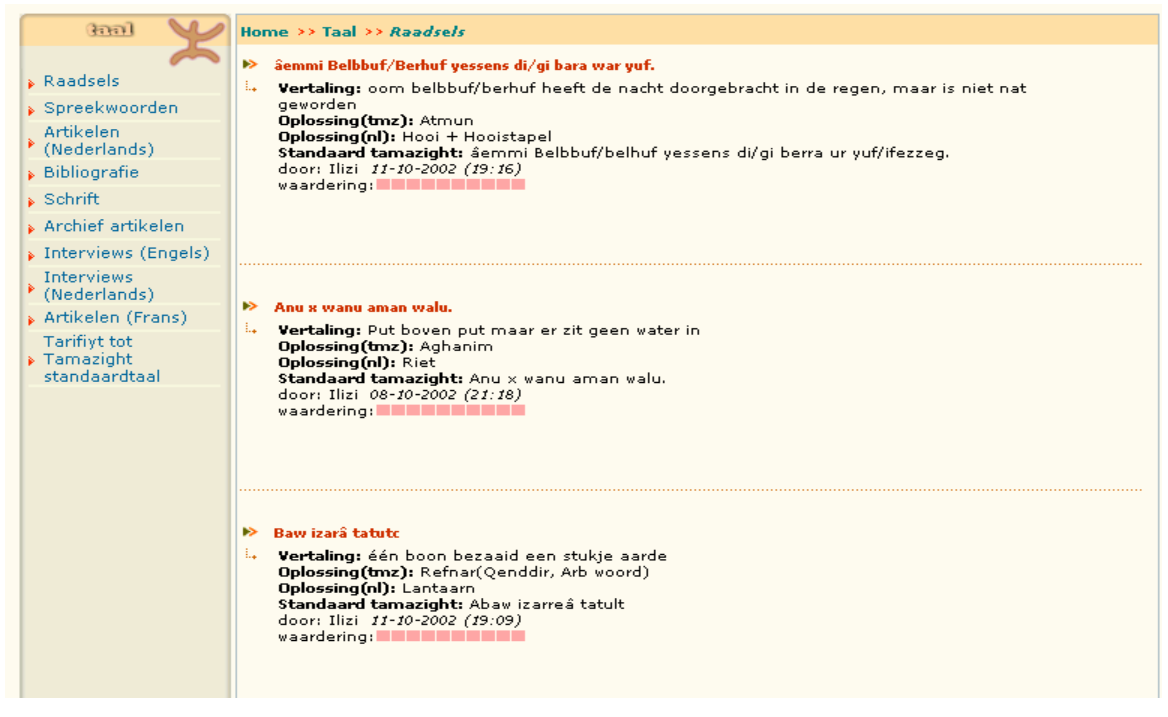

\author{
Extrait de devinettes du site Tawiza \\ (http://www.tawiza.nl/content/sectie.php?cid=48\&secties=cat)
}

Le tarifit occupe une position centrale dans la présentation de ces devinettes qui ont été rédigées en deux systèmes de notation à base latine.

La première et principale ligne de texte est écrite dans une notation que les éditeurs du site Tawiza emploient généralement de manière adéquate, mais toutefois sans donner aucune information à ce sujet. Ce manque d'information expliquerait pourquoi la plupart des textes présentés sur ce site sont rédigés dans une notation propre aux interactants, comme le montrent les expressions rifaines de l'exemple (01).

La seconde notation berbère (cinquième ligne de la devinette) est présentée comme la notation berbère «standard» (Standaard tamazight), bien qu'en réalité aucune notation unifiée ou «standard»-qu'elle soit fondée sur le latin, l'arabe ou le tifinagh (alphabet berbère ancien) - n'ait été conçue ni pour le tarifit comme une seule langue (ou variété) ni pour les langues berbères dans leur ensemble. Son étiquetage comme «standard » exprime l'aspiration des éditeurs du site Web de transmettre ces devinettes - produites oralement initialement - dans un format culturel «moderne » incarné par l'écrit et l'homogénéité linguistique.

La présentation du tarifit comme une langue «écrite» et «standardisée » non seulement répond à la demande et la pression omniprésentes de « modernité » mais rend cette langue aussi plus puissante au plan interactif par le relèvement de son statut social et culturel. Le choix d'une notation à base latine renforce ce statut parce qu'elle est communément considérée, dans le monde universitaire aussi bien que dans les milieux militants, comme l'option la plus viable et «moderne» pour écrire le berbère. Néanmoins, l'alphabet tifinagh est un concurrent de plus en plus important dans le débat sur la notation berbère (Lafkioui, 2002) . $^{8}$

8 Le fait que l'IRCAM (Institut Royal de la Culture Amazighe) ait opté pour l'alphabet tifinagh pour leurs pratiques d'alphabétisation a eu un certain impact sur l'orientation de la dynamique de l'orthographe des langues berbères au Maroc. 
Il est remarquable de constater que le transfert de ce genre littéraire d'un contexte interactif oral et local vers un contexte digital et translocal n'affecte pas sa fonction sociale et culturelle primaire, à savoir la transmission et l'échange de la tradition culturelle berbère. L'ensemble de l'imaginaire symbolique que ces expressions évoquent et requièrent afin d'être comprises fait également partie de cette tradition littéraire (Lafkioui, 2008a, 2008b).

\section{RELOCALISATION DIGITALE DE LA POÉSIE ORALE}

Dans cette section, j'examinerai comment la poésie orale et, plus précisément, son contexte de performance, peut être reconstruit par le biais d'Internet. Les deux exemples suivants (03 et 04), extraits du site Web Tawiza, montreront comment la poésie orale berbère peut être relocalisée en remplaçant son contexte traditionnel, c'est-à-dire des échanges verbaux entre le/la poète et une audience physiquement présente dans un espace déterminé à un moment précis, par un contexte digital contenant du texte écrit qui peut être lu à tout moment par tout internaute visitant le site. 


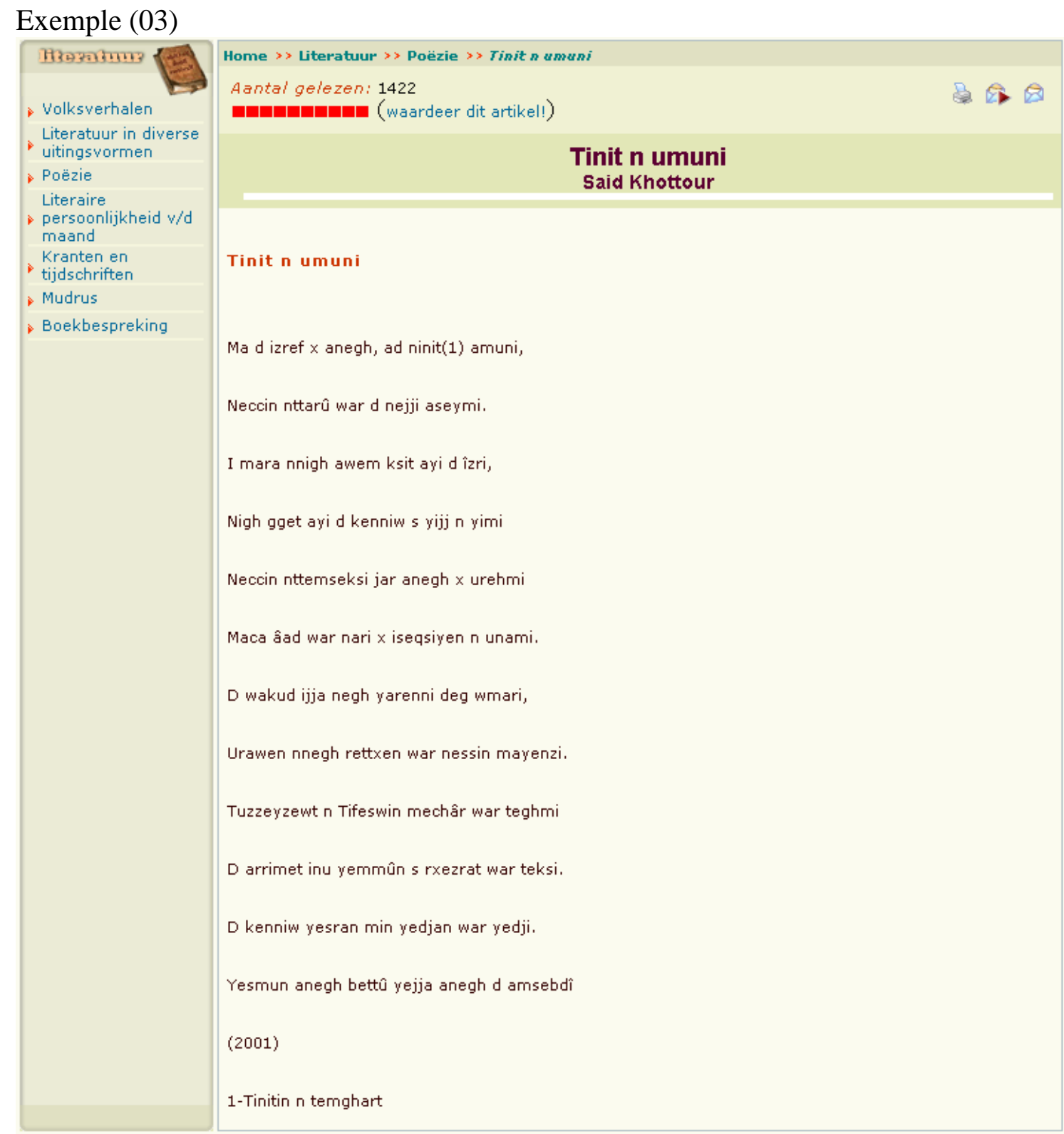

Said Khottour (2001) - DV, 11-09-2003 (20.42) sur Tawiza (http://www.tawiza.nl/content/awid.php?id=435\&sid=1\&andra=artikel)

Le poème Tinit $n$ umuni ('Le désir de la compagnie', exemple 03) est présenté sur la sous-page « Poësie » ('Poésie') de l'Hyperlink «Literatuur » ('Litérature') sans aucune traduction ou information ${ }^{9}$. Son auteur est un militant berbère qui a quitté le Maroc à un âge plus avancé, et ce avec une certaine connaissance et affection pour la langue et la culture rifaines ${ }^{10}$. Les productions littéraires (poèmes, expressions, devinettes, contes, etc.) de ce genre de poète sont généralement composées en tarifit et souvent suivies d'une traduction en néerlandais, comme le montre aussi l'exemple (04) provenant d'un autre poète.

9 A l'exception de la note de bas de page (1) qui essaie d'expliquer le syntagme verbal ad ninit (nous désirerons).

10 J'ai obtenu ces informations par le biais d'un entretien direct avec l'auteur. 


\section{Exemple (04)}

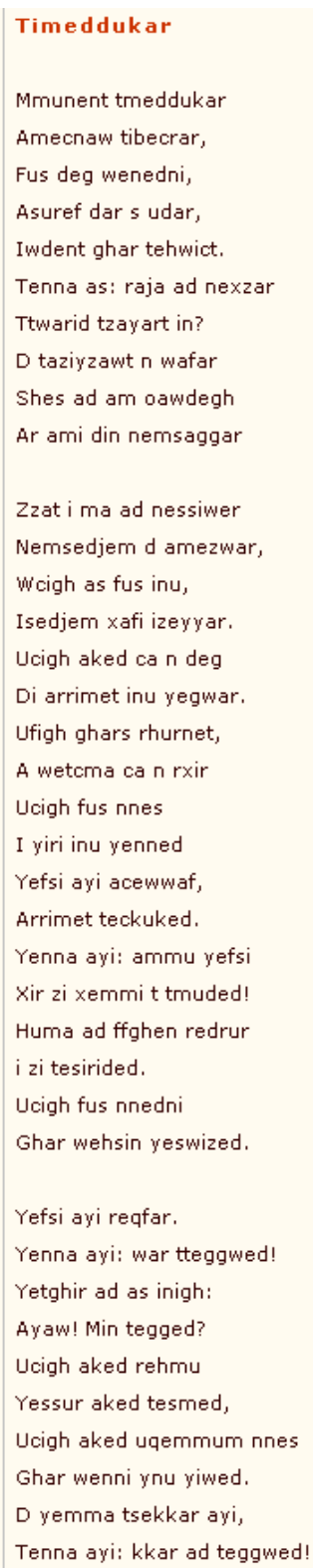

\begin{tabular}{|c|c|}
\hline \multirow{2}{*}{\multicolumn{2}{|c|}{$\begin{array}{l}\text { Yriendinnen } \\
\text { De twee vriendinnen vergezelden elkaar, } \\
\text { Samen, één paar. } \\
\text { Hand in hand } \\
\text { De ene stap na de andere, } \\
\text { Tot zij bij de tuin aankwamen. } \\
\text { Zij zei tegen haar: wacht even, } \\
\text { Zie je die wingerd, } \\
\text { Met zijn blauwe bladeren? } \\
\text { Luister, ik zal je vertellen, } \\
\text { Toen wij elkaar daar ontmoeten. }\end{array}$}} \\
\hline & \\
\hline & $\begin{array}{l}\text { Voordat we met elkaar spraken, } \\
\text { Begroetten we elkaar. } \\
\text { Ik gaf hem mijn hand, } \\
\text { welke hij stevig pakte. } \\
\text { Ik voelde iets vreemds } \\
\text { Door mijn lichaam gaan. } \\
\text { Ik voelde me rustig, } \\
\text { Mijn zuster, wat een gevoel. } \\
\text { Ik voelde zijn hand } \\
\text { Om mijn nek. } \\
\text { Hij maakte mijn haar los, } \\
\text { er ging een rilling door mijn lijf. } \\
\text { Hij zei: Zo, los staat je beter } \\
\text { dan gevlochten. } \\
\text { De kruiden waarmee } \\
\text { je je haar wast zullen opstijgen. } \\
\text { Ik voelde de andere hand } \\
\text { naar mijn buik reiken. }\end{array}$ \\
\hline & $\begin{array}{l}\text { Hij ontknoopte een knoop, en zei: niet bang zijn! } \\
\text { Hij dacht dat ik zou zeggen: } \\
\text { Pas op! Wat doe je? } \\
\text { Ik voelde warmte } \\
\text { en kou tegelijk. } \\
\text { Ik voelde zijn mond } \\
\text { Naar de mijne komen. } \\
\text { Totdat mijn moeder mij wekte, } \\
\text { En zei: Sta op en ga brood kneden! }\end{array}$ \\
\hline
\end{tabular}

Ahmed Ziani - DV on 21-11-2004 (23.14) sur Tawiza

Extrait de la collection Ighembab yarezzun $x$ wudem nsen deg wudem $n$ waman

Traduction néerlandaise : K. Awragh (http://www.tawiza.nl/content/awid.php?id=576\&sid=1\&andra=artikel) 
Ziani est un poète bien connu dans le mouvement culturel rifain. Il fait partie de la première génération de Rifains en Europe qui essaient de promouvoir la culture berbère par la poésie. Au cours de son long séjour aux Pays-Bas, il a assisté et participé à l'essor et la diversification de la production littéraire rifaine contemporaine. Conformément aux coutumes de sa génération, il a créé et présenté sa poésie d'abord oralement, et ce exclusivement en berbère. C'est seulement avec l'introduction de la pratique de noter les poèmes oraux (généralement en caractères latins) depuis les vingt dernières années que les poètes rifains se sont intéressés à la traduction ${ }^{11}$.

La poésie écrite rifaine et sa traduction en néerlandais ont connu un élan considérable avec l'arrivée des sites Web berbères à base néerlandaise. Ces derniers offrent non seulement des moyens plus rapides, captivants et bon marchés pour (re)publier des textes mais les rendent aussi accessibles à un lectorat potentiel plus étendu. Sur ces sites Web «minoritaires », l'écrit et la traduction occupent une position prépondérante pour des raisons interactives et translocales.

Contrairement aux deux poèmes précédant (exemples 03 et 04 ), le poème Tijd ('Temps') de l'exemple (05) est composé pour une publication digitale. L'absence du nom de l'auteur et de la date de publication confirme que ce poème n'a pas déjà été publié hors ligne. Au-delà du fait que l'auteur a choisi de publier sa poésie sur un site berbère, l'emploi du pseudonyme artistique berbère Tilmasin marque fortement son choix identitaire. Ce nom d'homme est écrit selon une des notations usuelles du tarifit.

Avec ce poème, l'auteur appelle à la reconnaissance et la préservation de l'identité rifaine, et ce avec une certaine mélancolie pour le passé et une grande adoration pour les combattants de résistance berbères.

Exemple (05)

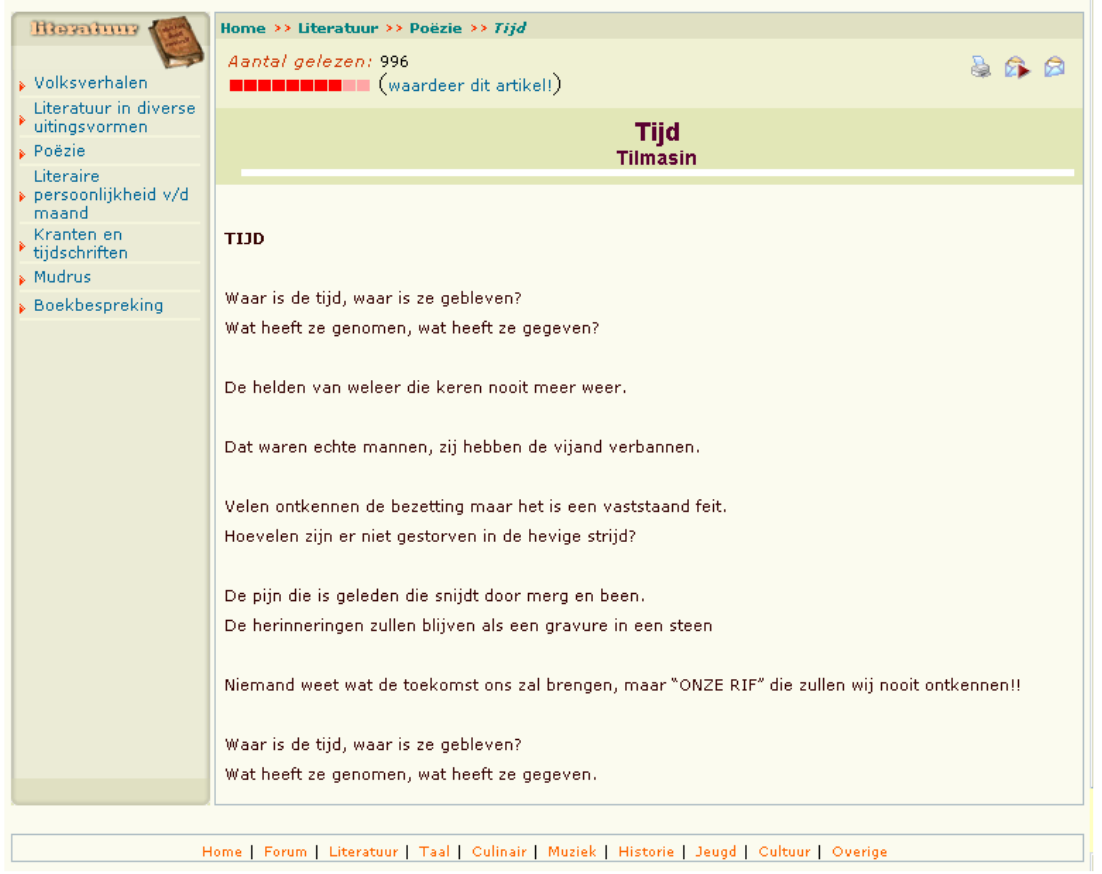

Tilmasin - DV, 02-07-2003 (23.49) sur Tawiza

(http://www.tawiza.nl/content/awid.php?id=430\&sid=1\&andra=artikel)

11 Il m'importe de signaler la contribution importante de Roel Otten à la traduction et la publication de la production littéraire rifaine aux Pays-Bas. 
Bien que la description affective de la résistance ne comporte pas de précisions historiques dans ce poème ('Temps'), la référence au mouvement de résistance des tribus rifaines contre les colons espagnols et français au début du vingtième siècle serait la plus vraisemblable pour ceux qui connaissent l'histoire récente du Rif (savoir commun comme contexte).

Cependant, diverses autres interprétations sont possibles, grâce à l'absence d'indication temporelle. L'occupation arabo-musulmane de l'Afrique du nord, qui a fini par supplanter des domaines variés de la culture berbère par sa nature et sa structure hégémoniques (domination culturelle), pourrait aussi être une interprétation appropriée car elle expliquerait l'énoncé :

Velen ontkennen de bezetting maar het is een vaststaand feit.

'Plusieurs nient l'occupation, mais c'est un fait accompli.'

La guerre de résistance du Rif contre l'occupation occidentale est un fait historique bien connu au Maroc. En effet, elle contribue encore, de nos jours, à l'identité nationale marocaine qui avait besoin d'être reconstruite après l'indépendance, et ce comme une alternative opposée à l'hégémonie des colons occidentaux. De ce fait, l'occupation la plus probable à laquelle renvoie le poète serait celle des Arabes musulmans; elle a perdu sa catégorisation sociale de «colon » avec le temps à cause des effets d'amnésie que son hégémonie idéologique exerce. Le caractère tout-englobant et coercitif de l'idéologie arabo-musulmane rend sa présence imperceptible pour les adhérents à son système (Barthes, 1957).

Ce poème digital pourrait être donc une voix translocale exprimant un désaccord avec certaines idéologies. Mais il pourrait, toutefois, aussi correspondre à de nombreux autres signifiés car sa contextualisation digitale et translocale élargit ses interprétations potentielles de manière considérable.

L'expression emphatisée «ONZE RIF » ('notre Rif') de l'extrait suivant réfère à un espace interactif spécifique correspondant à un espace physique - l'aire géographique du Rif dans laquelle des pratiques interactives ont lieu - autant qu'à un espace cognitif composé de croyances historiques, sociales, culturelles et idéologiques.

Niemand weet wat de toekomst ons zal brengen, maar "ONZE RIF” die zullen wij nooit ontkennen!!

'Personne ne sait ce que le futur nous apportera, mais «NOTRE RIF » nous le nierons jamais !!'

Du fait de sa nature translocale, le contexte digital (le site Tawiza) non seulement fait ressortir les aspects cognitifs de cette expression mais favorise aussi la (re)construction de l'identité collective berbère. Pour des raisons de repositionnement du Soi et de l'Autre dans l'espace interactif global, le translocalisme (transnationalisme) constitue un élément mobilisateur important pour la reconstruction des identités collectives (Vertovec, 2001). De la sorte, l'identité translocale rifaine a été relocalisée dans ce poème digital qui est incorporé dans un contexte local néerlandais, représenté essentiellement par l'usage de la langue néerlandaise sur le site berbère Tawiza (Appadurai, 1991, 1995 ; Meyrowitz, 2005). 
Le marqueur déictique onze ('notre') indique que l'auteur/interactant s'identifie avec l'identité collective orthodoxe rifaine correspondant à des représentations spécifiques sur la langue, la culture et l'histoire. L'emploi de cette expression révèle également un important engagement subjectif de la part de l'auteur par rapport à son discours. En fait, tout le poème dévoile un degré élevé d'investissement discursif de la part du poète.

\section{CONSTRUCTION IDENTITAIRE PAR INTERACTION DIGITALE SUR DES « CHAT FORUM »}

Les processus de construction identitaire se laissent particulièrement bien observer sur les sites Web berbères dans les «chat forum » (forums de discussion; Lafkioui, 2008b). Ainsi, j'ai pu constater que les représentations linguistiques des interactants exprimées par leurs interactions digitales sur ces forums sont fortement reliées à leurs histoires et idéologies sociales et individuelles. L'extrait suivant du site berbère Tawiza le confirme (exemple 06). Il présente une discussion digitale entre Yidir (masculin) et Drifa (féminin), deux noms qui sont fort probablement des pseudonymes ${ }^{12}$.

Exemple (06)

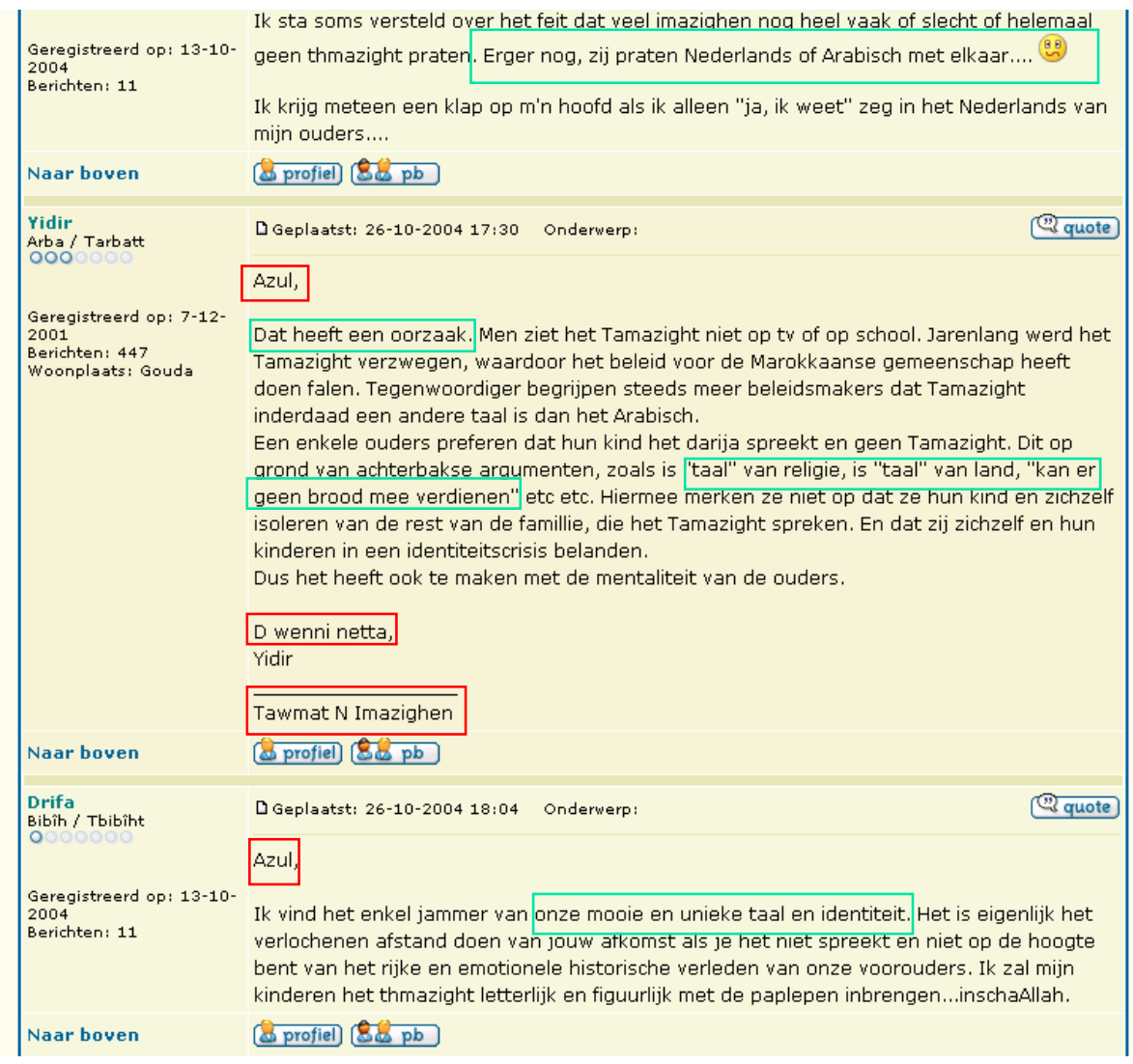

Extrait d'interaction chat forum sur Tawiza

12 A propos de l'usage des pseudonymes sur les sites de chat, voir Béchar-israélien (1995). 
Drifa ouvre la discussion en exprimant son étonnement sur le défaut de connaissances et compétences du tarifit chez les jeunes berbères aux Pays-Bas. De ses paroles ressort clairement que ses parents surveillent strictement sa performance et ainsi aussi sa compétence en rifain. Elle exprime également sa désapprobation de l'usage du néerlandais ou de l'arabe dans les interactions entre berbérophones, bien qu'elle utilise elle-même le néerlandais pour son échange digital avec son interactant berbérophone Yidir :

\section{Erger nog, zij praten Nederlands of Arabisch met elkaar... sad emoticon}

'Pire encore, ils parlent le néerlandais ou l'arabe entre eux... emoticon triste'

Son attitude négative à l'égard de cet état de choses est affichée par l'expression erger nog ('pire encore') et l'emoticon triste qu'elle a choisi comme clôture à son jugement de valeur. Son choix lexical implique une recatégorisation de l'objet de discours (sujet de discussion - forum topic), qui est Wie praat er Thmazight met z'n/haar ouders? ('Qui parle tamazight avec ses parents ?'). Par son choix lexical, Drifa s'engage fortement par rapport à son discours et augmente ainsi la potentialité de tension interactive entre elle et son interactant digital Yidir.

Cependant, l'expression de cette prise de position (stance) n'entraîne pas en fait un sérieux risque de conflit entre les interactants car un consensus de base est assuré par le site Web berbère qui fonctionne comme attributive centring institution (Silverstein, 1998) et comme gate-keeping apparatus (Gumperz, 1982) régulant dans une certaine mesure les propriétés du langage, ses fonctions et sa contextualisation ${ }^{13}$. La plupart des personnes qui participent aux forums sur ces sites adhèrent plus ou moins aux idéologies militantes berbères (group-specific ideologies), comme le confirment les expressions emblématiques berbères, telles Azul ('Salut !'), D wenni nta ('Au revoir !') et en particulier l'expression Tawmat $N$ Imazighen ('Fraternité berbère') employée par Yidir comme une sorte de signature à la fin de sa réponse. La composante Tawmat ('fraternité') de cette dernière expression révèle une référence sémantique et pragmatique implicite aux valeurs translocales mais subculturelles très ancrées dans le concept fort médiatisé de «fraternité afro-américaine ».

Le fait que Drifa fasse ici exactement ce qu'elle désapprouve, c'est-à-dire l'usage du néerlandais avec un berbérophone, suggère un écart entre ses représentations du tarifit et ses pratiques langagières in vivo. Le tarifit en tant que ressource digitale assume ici la fonction interactive de marqueur symbolique - l'icône - de l'identité collective berbère. En revanche, la fonction instrumentale de la langue - généralement remplie par des langues interactivement «dominantes » dans les contextes hors ligne - est attribuée au néerlandais.

Dans sa réponse, Yidir formule son accord avec Drifa sur la «perte » de la langue maternelle berbère au sein des jeunes, mais tente toutefois aussi de l'expliquer : Dat heeft een oorzaak ('Il y a une cause pour cela'). Il l'attribue à des facteurs sociaux, historiques et politiques, et ce avec une certaine autorité accordée par lui-même à son discours. A son avis, ceux qui sont aussi à blâmer pour cette situation particulière sont les parents qui préfèrent enseigner darija ('arabe dialectal', en l'occurrence, l'arabe marocain) à leurs enfants pour des raisons religieuses ("taal" van religie - "«langue » de la religion'), idéologiques ("taal” van land - "«langue» du pays ${ }^{14}$ ) et/ou

13 Dans une certaine mesure, le contexte digital permet également d'éviter le risque de perte de face des interactants (voir Goffmann, 1967 pour la notion de « face »).

14 La notion de « pays » renvoie ici à la signification de «pays d'origine » et donc de « patrie ». 
économiques ("kan er geen brood mee verdienen" - " "on ne peut pas gagner son pain avec »'). Ce sont eux qu'il juge responsables de la crise d'identité qu'ils subissent et qu'il font subir à leurs enfants.

Drifa répond dans le troisième paragraphe qu'elle regrette cette dégradation de onze mooie en unieke taal en identiteit ('nos belles et uniques langue et identité'). Le marqueur déictique onze ('notre') montre que Drifa s'identifie à l'identité collective berbère orthodoxe comprenant des représentations spécifiques sur la langue, la culture et $l^{\prime}$ histoire ${ }^{15}$. Son adoption de cette identité implique un certain rejet de l'usage des langues non berbères en présence de personnes berbérophones. Car pour Drifa, adopter l'identité berbère signifie l'assimilation de sa langue et de son histoire, comme indiqué dans la citation suivante en néerlandais :

Het is eigenlijk het verlochenen afstand doen van jouw afkomst als je het niet spreekt en niet op de hoogte bent van het rijke en emotionele historische verleden van onze voorouders. Ik zal mijn kinderen het thmazight letterlijk en figuurlijk met de paplepen inbrengen... inschaAllah.

'C'est, en fait, réfuter (et) renoncer à ton origine si tu ne la parles pas et si tu n'es pas au courant du passé historique riche et émotionnel de nos ancêtres. Moi, je nourrirai mes enfants avec du berbère à la cuillère au sens propre et figuré... si Dieu le veut.'

Ainsi, la langue berbère est une composante définitoire essentielle de l'identité collective berbère, construite en opposition à l'hégémonie étatique, que ce soit en Afrique du nord ou dans la diaspora. La notion de communauté berbère est fortement présente dans les discours en ligne et hors ligne actuels des militants berbères parce que l'idée de communauté linguistique augmente la capacité de créer des identités collectives (Silverstein, 1998).

Cependant, les interactions digitales in vivo sur les sites Web berbères à base néerlandaise et leurs pratiques de construction identitaire correspondantes sont pour la plupart bilingues ou multilingues. Les personnes qui participent à ces interactions, en partageant les ressources digitales requises, constituent donc une communauté de langage (speech community), un concept qui se distingue de celui de communauté linguistique ou communauté de langue (language community) qui indexe le fait que les interactants estiment qu'ils parlent et/ou écrivent la même langue (Silverstein, 1998).

Comme le montre bien l'exemple (07) qui suit, l'activité de « chatting » permet aussi de recontextualiser (méta-)discursivement les instances interactives, telles que des textes oraux et écrits, et ce par le biais de la nature réflexive de la langue (Lafkioui, 2008b) ${ }^{16}$.

15 Drifa s'implique fortement dans son discours en utilisant cette expression déictique. En fait, l'ensemble de l'extrait révèle un degré élevé d'engagement discursif de la part de l'interactant.

16 A propos de la nature réflexive de la langue, voir Rampton (2001) et Verschueren (2001). 
Exemple (07)

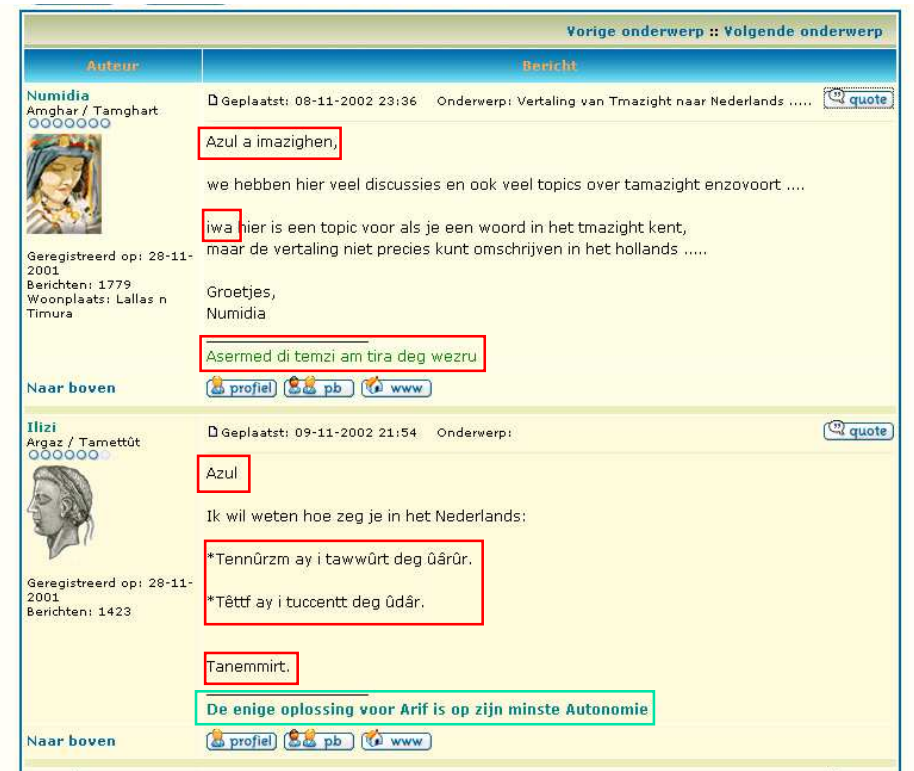

Extrait d'interaction chat forum sur Tawiza

Dans cet extrait, Ilizi demande comment traduire en néerlandais ces deux expressions berbères :

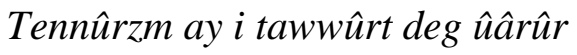

Littéralement : 'Une porte a été ouverte dans mon dos.'

$\Rightarrow$ Expression employée quand on sent tout à coup une douleur aiguë dans le dos.

Têttf ay i tuccentt deg $\hat{u} d \hat{r} r$

Littéralement : 'Le chacal femelle m'a pris par le pied.'

$\Rightarrow$ Expression employée quand on sent tout à coup une crampe au pied.

Dans ces interactions, les pratiques de code-switching entre le rifain et le néerlandais expriment des représentations du Soi et de l'Autre. Des témoignages d'identité encore plus explicites sont les «signatures » qui figurent à la fin de chaque échange :

Asermed di temzi am tira deg wezru (tarifit)

'Apprendre jeune, c'est comme la gravure sur pierre. ${ }^{, 17}$

De enige oplossing voor Arif is op zijn minste Autonomie (néerlandais)

'La seule solution pour le Rif, c'est du moins son autonomie.'

Toutes les deux prises de position sont fort émotives mais celle formulée en rifain contient tout de même une plus grande charge symbolique et expressive du fait du code linguistique utilisé.

17 C'est à la compétence du rifain que réfère cet énoncé. 


\title{
CONCLUSION
}

Internet contribue, de manière essentielle, au passage de l'oral à l'écrit des langues à tradition orale. Il permet aussi de diversifier l'oralité - oralité multimodale - comme production culturelle et métaculturelle et la rend accessible au monde. Des présentations de poésie, des interviews, des films, des spectacles de danse, entre bien d'autres, peuvent être écoutés et visualisés dans leur forme digitale par tout un chacun connecté à Internet. Bien que la valorisation de certains genres et productions littéraires traditionnels sur les sites Web «minoritaires » puisse avoir comme objectif principal la revendication et la construction identitaires, leur reproduction comme composantes du «patrimoine» culturel correspond aux discours idéologiques hégémoniques d'exclusivité culturelle et de modernité (Kirshenblatt-Gimblett, 2004).

Au cours de ces interactions bilingues/multilingues de type chat forum, un consensus de base est généralement atteint entre les interactants sur le contenu de leur discours ainsi que sur les positions de l'espace interactif qu'ils occupent pour s'exprimer. Le contexte digital permet donc cette négociation des identités conjointement (re)créées des représentations du Soi et de l'Autre - à partir de positions interactives symétriques et relativement plus convenables.

Cependant, les sites Web berbères sont des contextes institutionnalisés qui regroupent différents genres institutionnalisés au sein d'un «institutional discursive regime » (Fairclough, 1992) translocal plus large. Les sites Web berbères constituent des fora discursifs en plein essor qui recueillent des idéologies collectives spécifiques ; certaines d'entre elles sont visibles comme marqueurs symboliques d'identité collective, d'autres, en revanche, demeurent invisibles en raison de leur nature tout englobant. Internet est donc un instrument de pouvoir contenant des symboles puissants. Le transfert de ces multiples symboles à travers Internet offre un énorme potentiel sémiotique pour la construction et la reconstruction des identités collectives.

\author{
Mena LAFKIOUI \\ Universiteit Gent \\ Università di Milano-Bicocca
}

\section{RÉFÉRENCES BIBLIOGRAPHIQUES}

ANDROUTSOPOULOS, J. 2006a. Introduction: Sociolinguistics and computer-mediated communication. Journal of Sociolinguistics, 10/4: 419-438.

ANDROUTSOPOULOS, J. 2006b. Multilingualism, Diaspora, and the Internet: Codes and identities on German-based Diaspora websites. Journal of Sociolinguistics, 10/4: 520-547.

APPADURAI, A. 1991. Global Ethnoscapes. Notes and Queries for a Transnational Anthropology. In: R. Fox (ed.), Recapturing Anthropology, Santa Fe: School of American Research Press, 191-210.

APPADURAI, A. 1995. The production of locality. In : R. Fardon (ed.), Counterworks: Managing the diversity of knowledge, Londen: Routledge, 204-225.

BARTHES, R. 1957. Mythologies. Paris: Le Seuil. 
BECHAR-ISRAELI, 1995. From <Bonehead> to <cLoNehEAd>: Nicknames, play and identity on Internet Relay Chat. Journal of Computer-Mediated Communication, 1 (2), retrieved from http://jcmc.indiana.edu/vol1/issue2/bechar.html.

Blackledge, A. \& PAVLENKo, A. 2002. Ideologies of language in multilingual contexts: Special issue. Multilingua, 21 (2/3).

Blommaert, J. 2005. Discourse. A critical introduction. Cambridge: Cambridge University.

BOURDIEU, P. 1982. Ce que parler veut dire. Paris: Fayard.

BouRdieu, P. 1991. Language and Symbolic Power. Oxford: Polity.

ENCREVÉ, P. 1982. A propos du «marché linguistique ». In : N. Dittmar \& B. Schlieben-Lange, La sociolinguistique dans les pays de langues romanes, Tübingen: Gunther Narr Verlag, 97-103.

EnCREVÉ, P. 2004. Langue et domination. In : L. Pinto \& al. (éds.), Pierre Bourdieu, sociologue, Paris: Fayard, 289-305.

ENCREVÉ, P. \& FORNEL, M. DE 1983. Le sens en pratique. Actes de la recherche en sciences sociales, 46 (1): 3-30.

FAIRClOUgh, N. 1992. Discourse and Social Change. Cambridge: Polity.

GAL, S. 1989. Language and political economy. Annual Review of anthropology, 18: 345-367.

GEORGAKOPOULOU, A. 1997. Self-presentation and interactional alignments in e-mail discourse: The style and code-switches of Greek messages. International Journal of Applied Linguistics, 7: 141-164.

GEORGAKOPOULOU, A. 2003. Computer-mediated communication. In: Verschueren, J., Östman, J.-O., Blommaert, J. \& al. (eds.), Handbook of Pragmatics, Amsterdam: Benjamins, 1-20.

GofFMAN, E. 1967. Interaction Ritual. Essays on Face-to-Face Behaviour. New York: Pantheon.

GOFFMAN, E. 1974. Frame Analysis: An Essay on the Organization of Experience. New York: Harper \& Row.

GOFFMAN, E. 1981. Formes of talk. Philadelphia: University of Pennsylvania.

GofFMAN, E. 1983. Felicity's Condition. American Journal of Sociology, 89, 1: 1-53.

GuMPERZ, J. 1982. Discourse Strategies. Cambridge: Cambridge University.

GuMPERZ, J. 1992. Contextualization revisited. In: Auer, P. \& Di Luzio, A. (eds.), The contextualization of Language, Amsterdam: Benjamins, 39-53.

GuMPerZ, J. \& HYMES, D. (eds), 1972. Directions in Sociolinguistics. Cambridge: Cambridge University.

HAMERS, J. F. \& BlanC, M. H. A. 2000. Bilinguality and Bilingualism. Cambridge: Cambridge University Press.

Heller, M. (ed.) 1988. Codeswitching: Anthropological and Sociolinguistic Perspectives. The Hague: Mouton.

HELLER, M. 1992. The politics of code-switching and language choice. Journal of Multilingual and Multicultural Development, 13: 123-142.

HELLER, M. 1995a. Language choice, social institutions, and symbolic domination. Language in Society, 24: 373-405.

HELLER, M. 1995b. Code-switching and the politics of language. In: Milroy, L. and Muysken, P. (eds), One speaker, two languages: Cross-disciplinary perspectives on code-switching, Cambridge: Cambridge University, 158-174.

HERRING, S. C. 1993. Gender and democracy in computer-mediated communication. Electronic Journal of Communication, 3: 1-17.

HERRING, S. C. 1996. Computer-Mediated Communication. Amsterdam: Benjamins.

HERRING, S. C. 1999. Interactional coherence in CMC. Journal of Computer-Mediated Communication, 4 (4), retrieved from http://jcmc.indiana.edu vol4/issue4/herring.html.

HOFFMAN, D. 1989. Language and culture acquisition among Iranians in the United States. Anthropology and Education Quarterly, 20: 118-132.

HYMES, D. 1966. Two types of linguistic relativity. In: Bright W. (ed.), Sociolinguistics: Proceedings of the UCLA Sociolinguistics Conference - 1964, The Hague: Mouton, 114-167.

HYMES, D. 1996. Ethnography, Linguistics, Narrative Inequality: Towards an Understanding of Voice. London, Taylor \& Francis. 
Kirshenblatt-Gimblett, B. 2004. Intangible Heritage as Metacultural Production. Museum International, 221-222 (vol. 56, $\mathrm{n}^{\circ}$ 1-2): 52-65.

LAFKIOUI, M. 1996. La négation en tarifit. In : S. Chaker \& D. Caubet (éds.), La négation en berbère et en arabe maghrébin, Paris: L'Harmattan, 49-77.

LAFKIOUI, M. 1998. Les Berbères et leur langue : le cas des immigrés berbères en Belgique, in: C. Canut (ed.), Attitudes, Représentations et Imaginaires en Afrique, Paris, L'Harmattan/Langues'O, 119-130.

LAFKIOUI, M. 2002. Le rifain et son orthographe : entre variation et uniformisation. In: Caubet D., Chaker, S. \& Sibille, J. (eds.), Codification des langues de France, Paris: L'Harmattan, 355366.

LAFKIOUI, M. 2006. Pratiques et représentations linguistiques en contexte multilingue. Le cas des Berbères en Belgique, Quaderni del Dipartimento di Linguistica (Unical), 24, 73-84.

LAFKIOUI, M. 2008a. Reconstructing Orality on Amazigh Websites. In: M. Lafkioui \& D. Merolla (éds.), Oralité et nouvelles dimensions de l'oralité. Intersections théoriques et comparaisons des matériaux dans les études africaines, Paris: Publications Langues'O, 111-124.

LAFKIOUI, M. 2008b. Identity construction through bilingual Amazigh-Dutch "digital" discourse, in: M. Lafkioui \& V. Brugnatelli (eds), Berber in contact: linguistic and sociolinguistic perspectives, Köln, Rüdiger Köppe Verlag, 217-231.

LAFKIOUI, M. \& MEROLLA, D. 2008. Oralité et nouvelles dimensions de l'oralité. Intersections théoriques et comparaisons des matériaux dans les études africaines. Paris: Publications Langues O'.

Merolla, D. 2005. Migrant Websites, WebArt, and Digital Imagination. In: S. Ponzanesi, S. \& Merolla, D. (eds.), Migrant Cartographies: New Cultural and Literary Spaces in Post-Colonial Europe, Oxford: Lexington Books, 217-228.

Merolla, D. 2008. Oral Genres in African 'Migrant' Websites. In : M. Lafkioui \& D. Merolla (éds.), Oralité et nouvelles dimensions de l'oralité. Intersections théoriques et comparaisons des matériaux dans les études africaines, Paris: Publications Langues O', 101-109. MEYROWITZ, J. 2005. The rise of glocality: New senses of place and identity in the global village. In: K. Nyiri (ed.), A sense of place: The global and the local in mobile communication, Vienna: Passagen Verlag, 21-30.

Pavlenko, A. 2002. Poststructuralist Approaches to the study of social factors in second language learning and use. In: Cook V. (ed), Portraits of the L2 user, Clevedon: Multilingual Matters, 277-302.

RAMPTON, B. 1995. Crossing. Language and ethnicity among adolescents. London/New York: Longman.

RAMPTON, B. 2001. Critique in interaction. Critique of Anthropology, 21: 83-107.

SiLVERSTEIN, M. 1998. Contemporary transformations of local linguistic communities. Annual Review of Anthropology, 27: 401-426.

VERSCHUEREN, J. 2001. Predicaments of criticism. Critique of Anthropology, 21: 59-81.

VERTOVEC, S. 2001. Transnationalism and identity. Journal of ethnic and migration studies, 27 (4): 573-582.

WILliaMS, G. 1992. Sociolinguistics : a Sociological Critique. London: Routledge. 